\title{
An Improved Track Initial Algorithm Based by Waveform Agility
}

\author{
Ren Li \\ University of Electronic Science and Technology of \\ China \\ Chengdu ,China \\ renl@uestc.edu.cn
}

\author{
Yang Feng \\ University of Electronic Science and Technology of \\ China \\ Chengdu ,China
}

\begin{abstract}
Currently tracking Track initiation in most core processes, how to achieve multiple objectives in a complex environment more accurate track initiation is still the key and difficult problems of target tracking. Waveform agile radar is currently the latest breakthroughs in the field of cognitive technology radar aspect, it is different from the conventional radar characteristics are mainly from the environment (including the target) in continuous learning to improve target detection capability. This paper introduces minimum gate volume to logic track initiation algorithm, which thesis proposes a track initiation agility algorithm by controlling gate volume to reduce amount of clutter, which improves success rate and accuracy of track.
\end{abstract}

Keywords-Waveform agility; Covariance control; Data rate; Joint agility; Track initial.

\section{INTRODUCTION}

Complex Environment radar multi-target tracking technology is facing serious challenges in the clutter density, the number of the target number of cases, the current multitarget tracking (MTT) technology research focused on two key points: technology and multi-target data association filtering algorithm, the corresponding theory and techniques have been more perfect. But still cannot completely solve the complex environment of multi-target tracking problem issues in this area is one of academia active direction.

The recent rise of the waveform agility techniques already been applied in a single-target detection and tracking, then the waveform agility technology to maintain a desired multi-target tracking performance and increased track capacity is extremely interesting research topic. Waveform agility is a multi-target tracking another hot issue worth exploring, is conducive to sustained stable and efficient multi-target tracking. Research on real-time waveform agile and efficient method for maintaining a desired multi-target tracking effect, a reasonable allocation of system resources is important.

Agile radar waveform using closed loop mode, adjust the transmit signal in real time according to the environment dynamic perception. When the system get more information about the scene (the target), and something about the target state estimation parameter is updated. After updating the system based on the scene (objective) knowledge of the transmitted waveform design, thereby increasing the probability of target detection, estimation accuracy, recognition accuracy, the tracking accuracy. [1]

The method of control theory was first used by Kershaw and Evans first proposed in 1994 [2], they use the system as the optimal tracking performance indicators waveform optimization. However, the algorithm ignores the external electronic interference, it can not be good in the presence of varying electronic jamming environment work.[3]

Paper 4 In order to improve the tracking performance of single targets, based on Bayesian framework, according to the posterior Cramer-Rao Bound (PRCB) the next time the transmitted waveform design and polarization.

Paper 5 through design OFDM signal waveform parameters to optimize detection performance to solve the OFDM signal in multipath environments for radar target detection problem.

By the above observation can be found, now waveform parameter agility tracking technology is mainly used to solve the problem of a single objective, research waveform parameter agility for multi-target tracking problems still in the initial exploratory stage, there is no a complete theory.

\section{TRACK INITIAL METHOD}

Target tracking is to track and measure the point target track for data associated with the associated trace points get updates the status of the target track. Multi-target tracking ships by the track initiation, related processing, tracking filtering step constituted, which track initiation is a key issue multiple target tracking.

Tracking gate is tracking the ownership of the decision of the measured value, which is the center point of the predicted value of the filter, the size of the gates is determined by the amount set in the real probability $P_{G}$ of falling into the door of the wave measured, $P_{G}$ is larger, the real the probability of the target measured value falls the greater, but at the same probability of false targets fall greater. Wave measured value falls inside the door, we call them the candidate echo, which measured value may include real targets and false measurement values, which are the basis of the data associated with the next data.

Here we have chosen the common gate spheroidal wave shape of the Cartesian coordinates. If the measured value acquisition radar satisfy [7]: 


$$
\begin{aligned}
& \tilde{\mathrm{V}}(\gamma)= \\
& {\left[\mathrm{z}_{c}(\mathrm{k}+1)-\hat{\mathrm{z}}_{c}(\mathrm{k}+1 \mid \mathrm{k})\right]^{T} S^{-1}(k+1)\left[\mathrm{z}_{c}(\mathrm{k}+1)-\hat{\mathrm{z}}_{c}(\mathrm{k}+1 \mid \mathrm{k})\right] \leq \gamma}
\end{aligned}
$$

Called conversion measured value as a candidate echo $\mathrm{z}_{c}(\mathrm{k}+1)$. Spheroidal wave equation becomes door rules, where the parameters $\gamma$ obtained from the $\chi^{2}$ distribution table. If the measured value $\mathrm{z}_{c}(\mathrm{k}+1)$ is $n_{z}$ dimension, $\tilde{\mathrm{V}}(\gamma)$ having $n_{z}$ degrees of freedom distributed $\chi^{2}$ random variables.

For different $\gamma$ values and different dimension $n_{z}$ measurement, different $P_{G}$, definitions:

$$
P_{G}=P\left\{\mathrm{z}_{c}(\mathrm{k}+1) \in \tilde{\mathrm{V}}_{k+1}(\gamma)\right\}
$$

Real probability measure falls dimensional spheroidal wave door as follows:

$$
P_{G}=1-(1+\gamma / 2) \exp (-\gamma / 2)
$$

$n_{z}$ Surface-dimensional ellipse (ball) gates (the body) volume of:

$$
\mathrm{V}\left(\mathrm{n}_{z}\right)=\mathrm{c}_{\mathrm{n}_{z}} \gamma^{\frac{\mathrm{n}_{z}}{2}}|S(\mathrm{k}+1)|^{\frac{1}{2}}
$$

Here:

$$
\mathrm{n}_{z}= \begin{cases}\frac{\pi^{\frac{\mathrm{n}_{z}}{2}}}{\left(\mathrm{n}_{z} / 2\right) !} & \mathrm{n}_{z} \text { is even } \\ \frac{2^{n_{z}+1}\left(\frac{n_{z}+1}{2}\right) ! \pi^{\frac{\mathrm{n}_{z}-1}{2}}}{\left(\mathrm{n}_{z}+1\right) !} & \mathrm{n}_{z} \text { is odd }\end{cases}
$$

\section{TRACK InITIAL METHOD BASED ON WAVEForm AGILITY}

Select state estimation is equivalent to making the smallest trace waveform parameter covariance matrix composition based on a minimum mean square error criterion cybernetics waveform agile method used as the next time the optimal transmit waveform, which called for the next time state-space dimensions square and minimal tracking error on; through the cost function is derived, was found based on information theory and cybernetics-based waveform agile methods are equivalent, agile waveform information theory-based approach by minimizing the state error covariance matrix estimate determinant to select the optimal transmit waveform.
To wave gate volume control and track initiation algorithm combining agile algorithm selects a logical track initiation algorithm, and select the oval gates Cartesian coordinate system, as relevant gates. Since the beginning of the first track requires two measurements to establish, and two differential filtering algorithm need to initialize, so in the third measurement started waveform parameters based on the criterion of wavelet door, designed in this paper based on the waveform agility track initiation process as shown below:

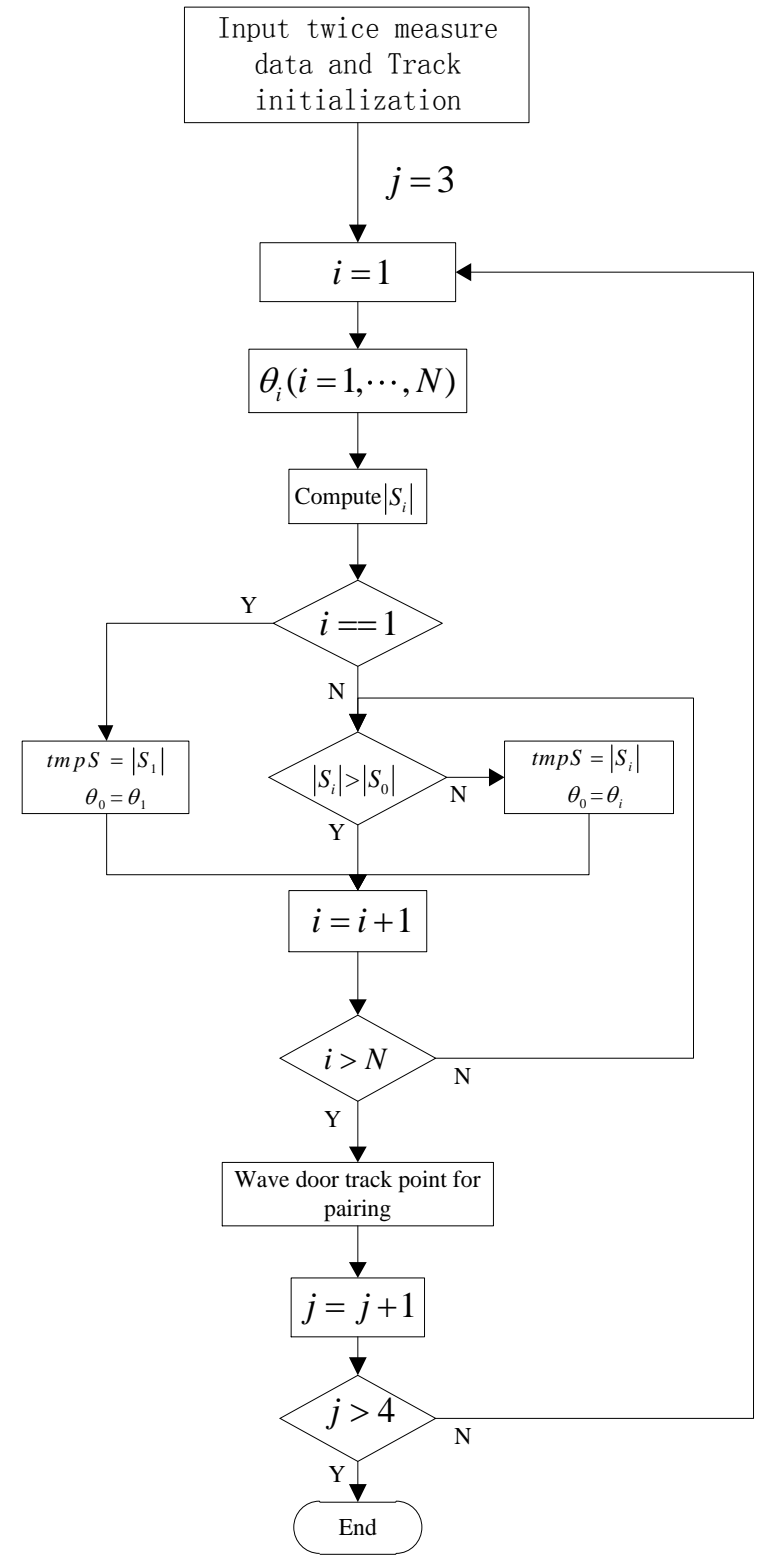

Figure 1. Starting flowchart algorithm based on the waveform agility $3 / 4$ logical track

Track initiation algorithm steps described below: 
1) Under the premise to meet the speed gates, according to the first two measurements to establish a track head.

2) The next forecast track the center position, and through the entire waveform libraries obtained gates makes the smallest combination of parameters.

3) Waveform parameters of the selected point in time according to the next track update, judging distance Prediction Center nearest point lies wave door, if the wave door, then make the appropriate filter processing; otherwise, the use of state and state prediction error covariance matrix filter updates.

4) Data sampling more than four times, and three times more than the corresponding number in the door a little wave trace do track correlation process, the initial success of the track, otherwise remove the track head. Sampling times is less than four, the process proceeds to step 2)

\section{Simulation}

To validate the algorithm, to build simulation scenario: Suppose three goals in the two-dimensional motion, the Earth's gravity model selection flat earth model, target model using the Singer model, confirm elliptical wave gate wave gate Cartesian coordinate system, using the traditional logical track initiation algorithm based on the wavelet door volume waveform parameter agility algorithms to a single time window (containing four data samples) of track initiation, agile waveform from the third sampling time.

Simulation parameters: the radar transmitter signal is Gaussian envelope chirp, carrier frequency 10.4CHE, beam width $0.5^{\circ}$, The data rate $1 \mathrm{~Hz}$ is fixed waveform parameters are: Pulse Width $T_{0}=40 e^{-6} s$, Frequency Rate $b_{0}=10 e^{8} s^{-2}$. The data rate $0.5 \mathrm{~Hz}$ is fixed waveform parameters are: Pulse Width $T_{0}=50 e^{-6} s$, Frequency Rate $b_{0}=30 e^{8} s^{-2}$.Agile waveform: Waveform pulse width I agility interval $\left[10 e^{-6} s, 1000 e^{-6} s\right]$, which is divided into 10 equal intervals; The maximum bandwidth $b w_{\max }$ limit $10 M H z$,

FM slope ${ }^{\gamma}$ agile interval $\left[-b w_{\max } / T_{\text {sel }}, b w_{\max } / T_{\text {sel }}\right]$, $T_{\text {sel }}$ is selected pulse width, which is divided into 10 equal intervals. Here we take Monte Carlo simulation 100 times.

Target motion model is Singer, which parameter is: Maneuvering Frequency $\alpha=1 / 60$, Maneuvering Acceleration $\sigma^{2}=0.001$. And a look-up table $\gamma=9$ according to $n_{z}=2$ and $P_{G}=0.989$. Wave velocity in the $x$ direction of the door $[80,120]$. Wave velocity in the $y$ direction of the door $[100,250]$. The initial state of the three objectives as follows:

$$
\begin{aligned}
& X 0=\left[\begin{array}{llllll}
10000 & 100 & 0 & 10000 & 200 & -0.98
\end{array}\right]^{\prime} \\
& X 1=\left[\begin{array}{llllll}
10400 & 100 & 0 & 10000 & 200 & -0.98
\end{array}\right]^{\prime} \\
& X 2=\left[\begin{array}{llllll}
10800 & 100 & 0 & 10000 & 200 & -0.98
\end{array}\right]^{\prime}
\end{aligned}
$$

Simulation initial scene shown below:

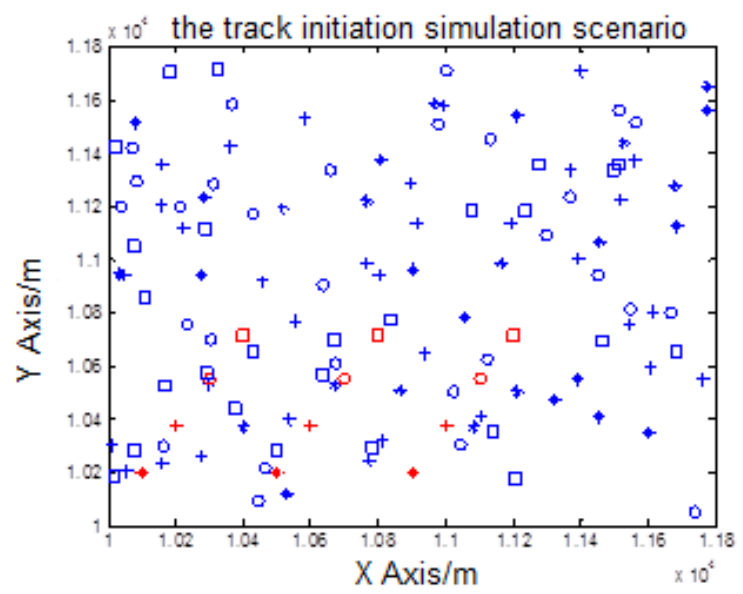

Figure 2. $1 \mathrm{~Hz}$. Data rate, $\lambda=30$ the track initiation simulation scenario

In order to obtain the image of the observed track initiation process of the two algorithms, Figure 2 and Figure 3 are selected 10th simulation, data rate. In Figure 2, respectively, and the first to fourth sampling time point mark, which clutter blue dot, red represents real measurement point

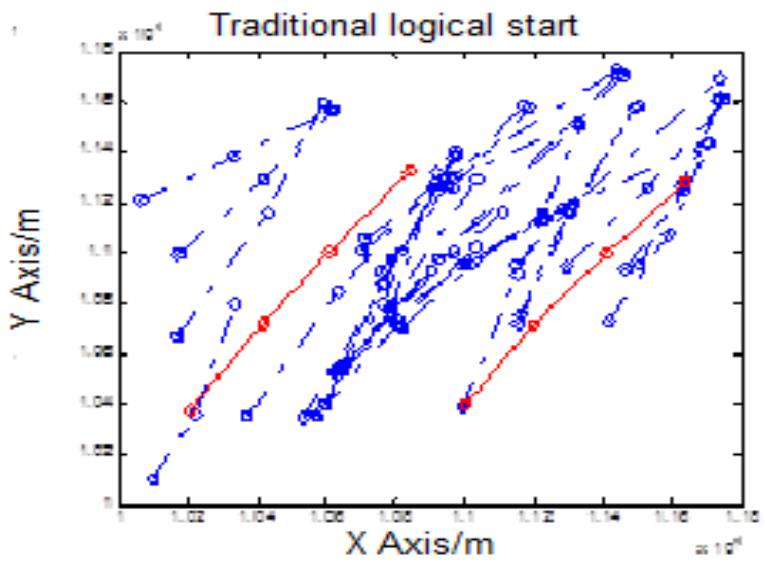

Figure 3. Traditional logical start 


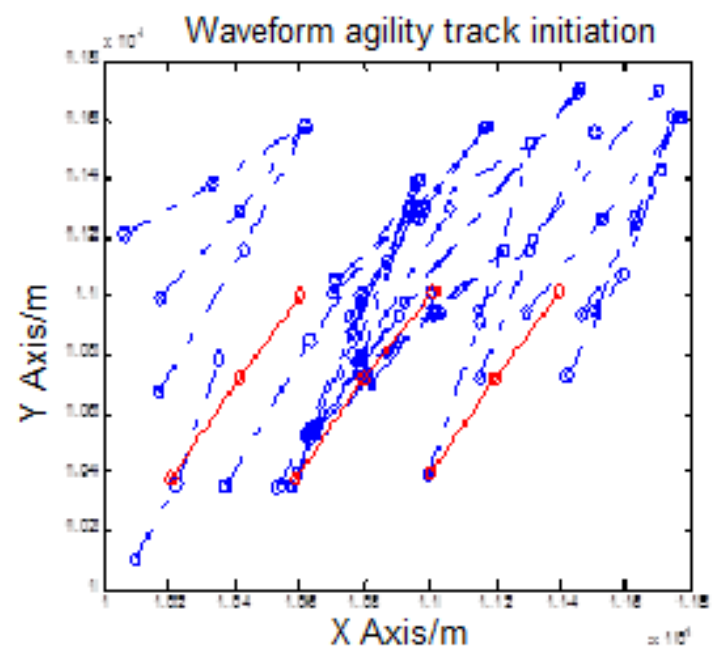

Figure 4. Waveform agility track initiation

Figure 3 and 4 blue dotted line indicates a successful start false track, the red solid line represents the start of a successful real track.

Table 1. Start TRack Performance Comparison of $1 \mathrm{~Hz}$ Data RATE

\begin{tabular}{|c|c|c|c|c|c|c|}
\hline \multirow{2}{*}{$\lambda$} & \multicolumn{3}{|c|}{ Fixed waveforms } & \multicolumn{3}{c|}{ Agile waveform } \\
\cline { 2 - 7 } & $\eta 1$ & $\eta 2$ & $t_{c}$ & $\eta 1$ & $\eta 2$ & $t_{c}$ \\
\hline 20 & $76 \%$ & $24.73 \%$ & 448.6 & $81 \%$ & $27.80 \%$ & 2032.7 \\
\hline 30 & $69 \%$ & $9.27 \%$ & 978.6 & $79.67 \%$ & $12.30 \%$ & 4047.9 \\
\hline 50 & $67.67 \%$ & $4.34 \%$ & 4037.6 & $77.67 \%$ & $5.51 \%$ & 11395.6 \\
\hline 70 & $60 \%$ & $1.94 \%$ & 9539.9 & $72.67 \%$ & $2.59 \%$ & 23712.9 \\
\hline
\end{tabular}

Here $t_{c}$ is the time consuming.

TABle 2. START TRACK PERFORMANCE COMPARISON OF $0.5 \mathrm{~Hz}$ Data RATE

\begin{tabular}{|c|c|c|c|c|c|c|}
\hline$\lambda$ & \multicolumn{3}{|c|}{ Fixed waveforms } & \multicolumn{3}{c|}{ Agile waveform } \\
\cline { 2 - 7 } & $\eta 1$ & $\eta 2$ & $t_{c}$ & $\eta 1$ & $\eta 2$ & $t_{c}$ \\
\hline 20 & $85.67 \%$ & $53.43 \%$ & 466.9 & $92.33 \%$ & $56.53 \%$ & 2101.9 \\
\hline 30 & $81.67 \%$ & $36.19 \%$ & 1038.8 & $90.67 \%$ & $37.23 \%$ & 3820.9 \\
\hline 50 & $80 \%$ & $13.54 \%$ & 3675.5 & $87.33 \%$ & $14.3 \%$ & 9685.9 \\
\hline 70 & $75 \%$ & $6.47 \%$ & 8670.8 & $79.33 \%$ & $6.42 \%$ & 19744.4 \\
\hline
\end{tabular}

By comparing Table 1 and Table 2 can be found through the waveform agility, the starting track the success rate of increase compared to the real target fixed waveforms are of the correct track initiation rates and some increase, but
Because agile algorithm needs to traverse each parameter combination calculated waveform agility required algorithm running time is about four times the fixed waveforms, and waveform parameters chosen is the maximum bandwidth and the maximum pulse width, so you need to consume more radar resources, but because only two times agility, and higher real success track initiation rates can reduce the average track starting time, so more of the cost of the radar resource consumption is acceptable. With the increase of noise density, the real target initial success rate and the starting rate showed a decreasing trend right track, this is because of the higher density of clutter will result in more false trajectory generation, so that the correct track number the proportion decreased.

\section{V.CONCLUSIONS}

Through the above analysis it can be concluded: the most agile waveform based on wavelet door track volume can effectively improve the success rate and the starting rate of correct track starting the real target, the corresponding cost of additional radar resource consumption, but for a successful start of the track, since only two agile process start-up phase, so the cost of agility is usually acceptable.

\section{REFERENCES}

[1] S. Haykin, A. Zia, Y. Xue, I. Arasaratnam. Cognitive Tracking Radar. Cognitive Systems Laboratory, McMaster University, 2009

[2] Kershaw D, Evans R. Optimal waveform selection for tracking systems. IEEE Trans on Information Theory, 1994, 40(5): 1536-1550

[3] Kershaw D, Evans R. Waveform selective probabilistic data association. IEEE Trans on Aerospace and Electronic Systems, 1997, 33(4): 1180-1188

[4] Hurtado M., Zhao T., Nehorai A.. Adaptive polarized waveform design for target tracking based on sequential Bayesian inference. IEEE transactions on Signal Processing, 2008, 56(3): 1120-1133

[5] D. Lerro, Y. Bar-Shalom. Automated Tracking with Target Amplitude Information[C]. American Control Conference, San Diego, USA, 1990, 2875-2880

[6] Sen S., Nehorai A.. OFDM MIMO radar with mutual-information waveform design for low-grazing angle tracking. IEEE transactions on. Signal Processing, 2010, 58(6): 3152-3162

[7] X. Rong Li, Vesselin P. Jilkov. Survey of Maneuvering Target Tracking. Part I: Dynamic Models. IEEE transactions on Aerospace And Electronic Systems, 2003, 39(4): 1333-1364 